\title{
Quality of Wax Esters, Alcohol and Cuticle Formation in Drought Tolerant and Drought Sensitive Wheats (Triticum aestivum) under Unfavourable Temperature and Radiation Conditions for Growth and Development
}

\author{
Santosh Kumari ${ }^{1 *}$ and Vipin Kumar Verma ${ }^{2}$ \\ ${ }^{1}$ Division of Plant Physiology, Indian Agricultural Research Institute, New Delhi, India \\ ${ }^{2}$ Department of Pharmacology, All India Institute of Medical Sciences, New Delhi, India \\ *Corresponding author
}

\section{A B S T R A C T}

Keywords

Cuticle, Drought, Fatty acids, Wax esters, Wheats

Article Info

Accepted:

08 January 2020

Available Online:

10February 2020

Very long chain fatty acids (C20 and longer) are synthesized via fatty acid elongases at ER. Plastid generated C16 and C18 fatty acids and malony CoA are substrates for these elongases. The cuticular waxes are a mixture of mostly aliphatic very long chain fatty acids (VLCFA) and variable amount of triterpenoids and phenyl propanoids. Cuticle is composed of inter-esterification of $\omega$ and mid chain hydroxy and epoxy C16 and C18 fatty acids and glycerol, and cuticular waxes. Our GC-MS data exhibit that VLCFA synthesis in drought tolerant wheat cultivar (C306) is indicator of retention of fatty acid synthesis and elongation ability in contrast to HD2428 drought sensitive wheat cultivar under drought due to unfavourable radiation and temperature.

\section{Introduction}

The plant cuticle provides protection from ultraviolet radiations, pathogen infections and regulates transpiration water loss and absorption. Cuticle is composed of interesterification of $\omega$ and mid chain hydroxy and epoxy C16 and C18 fatty acids and glycerol, and cuticular waxes. These waxes are a mixture of mostly aliphatic very long chain fatty acids (VLCFA) and variable amount of triterpenoids and phenyl propanoids. VLCFA precursors of cuticular waxes are synthesized in the epidermal cells by fatty acid elongases found on ER (endoplasmic reticulum) using plastid generated $\mathrm{C} 16$ and $\mathrm{C} 18$ fatty acids and malony $\mathrm{CoA}$ as substrates. B-Ketoacyl CoA synthase (KCS) is the rate limiting enzyme in the fatty acid elongation and determines the length of final acyl CoA product. Fatty acyl CoA reductase associated with peroxisomes produce fatty alcohols and wax synthase (ER associated) esterify these alcohols with acylCoA to wax esters. Very long chain fatty acids (C20 and longer) are synthesized via fatty acid elongases at ER by repeated cycles 
of two-carbon addition. The condensation of two carbons from malonyl-CoA to the growing acyl-CoA chain with release of $\mathrm{CO}_{2}$ and CoASH, followed by reduction using $\mathrm{NADPH}$, dehydration, and another reduction reaction, result in a saturated acyl-CoA. Termination of final reduction step may result in the formation of alkenes, aldehyde, alkanes, secondary alcohols and lactone formations.

Wax synthesis is induced by water deficit, sodium chloride and abscisic acid treatments. ABA is well established plant growth regulator under water stress. The plant cuticle is a defining feature of the epidermis; it plays a central role in development by physically establishing organ boundaries and restricting carbon utilization in plant biomass production. Wheat is global staple food, therefore, the cuticle component wax esters and fatty acids information will aid in understanding the photosynthates partitioning in plant biomass and grain production under unfavourable growth environment/ stresses. Wheat is exposed to salinity, drought, high temperature and radiations under late sown conditions in farmer's field. Therefore, GCMS [GCMS-QP2010 Plus] analysis was done for quality of wax esters, alcohol and cuticle formation in drought sensitive and drought tolerant wheats under unfavourable temperature and radiation conditions for growth and development.

\section{Materials and Methods}

Drought sensitive wheat cultivar, HD2428 and drought tolerant wheat cultivar- C306 plants were grown in green house in earthen pots (size $30 \times 30 \mathrm{~cm}$ ) filled with sandy loam soil and farmyard manure in 3:1 under natural environment (January 15, 2018). Each pot was fertilized corresponding to 120,90 and $60 \mathrm{~kg}$ ha- 1 of N, P and K, respectively. Plants were kept free from diseases. Flag leaves of wheats growing at $29^{\circ} \mathrm{C}$ unfavourable growth conditions were compared with flag leaves of control plants growing at $24^{0} \mathrm{C}$ (November 15, 2017).

Flag leaves at ear emergence stage were sampled and ground in liquid $\mathrm{N}_{2}$, homogenised in methanol, evaporated to dry powder and dissolved in methanol (HPLC grade) for GC-MS analysis. Photographic data was collected under normal as well as unfavourable growth conditions.

\section{Results and Discussion}

The physical and chemical constituents have been extensively investigated by chemists and microscopy. Epicuticular wax is the first layer in all terrestrial plants may be semi-crystalline or crystalline in structure. Thickness of this layer is changed by the environmental temperature and radiations. Cuticle is the layer beneath this first layer which is amorphous in nature.

Our data suggest that C16 type of cutin is predominant in rapidly expanding leaves growing at favourable growth temperature and radiation (Kolattukudy, 1980). Plants growing under unfavourable growth conditions produce in smaller leaves and constituents of cutin are governed by the susceptibility and tolerance of species to environmental stress

The composition of pigments i.e. carotenoids and chlorophylls are subjected to turnover by environmental factors to adapt the growth conditions. Chlorophyll cleavage release chlorophyllide and phytol during senescence.

Free phytol, a primary C20 isoprenoids alcohol (Methylerythritol pathway-MEP in plastids), accumulation destabilizes chloroplast/organelles membranes and their normal functions. 
Table.1 GC-MS analysis of wax esters, fatty acids, alcohols, aldehyde, alkanes, alkenes in flag leaves of drought tolerant and drought sensitive wheats under unfavourable temperature and radiation conditions for growth and development

\begin{tabular}{|c|c|}
\hline HD2428 (Control) - November & Drought - January \\
\hline Pentadecane & $\begin{array}{l}\text { 1-Tetradecene } \\
\text { Oxirane, tetradecyl- }\end{array}$ \\
\hline 1-Hexadecanol & 1-Hexadecanol \\
\hline 1-Octadecene & 2-Octyldodecan-1-ol \\
\hline Decanoic acid, 8-methyl-, methyl ester & Tetradecanoic acid, 12-methyl-, methyl ester \\
\hline Hexadecanoic acid methyl ester & Hexadecanoic acid methyl ester \\
\hline & $\begin{array}{l}* * \text { Heptadecanoic acid, methyl ester } \\
\text { Dodecanoic acid ethenyl ester (-Lauric acid vinyl ester) }\end{array}$ \\
\hline 9-Eicosene, $(\mathbf{E})-$ & 9,12,15-Octadecatrienal \\
\hline C306 (Control) & Drought - January \\
\hline & $\begin{array}{l}\text { 1-Tetradecene } \\
\text { 1-Heptadecene } \\
\text { 7-Oxanonadecane } \\
\text { Oxirane, tetradecyl- } \\
\text { Hexadecane 1,16-dichloro }\end{array}$ \\
\hline $\begin{array}{l}\text { 2-Hexadecen-1-ol, } \\
\text { 3,7,11,15-tetramethyl-, } \\
\text { [r-[r*,r*-(e)]]- (t-phytol }\end{array}$ & Phytol \\
\hline Hexadecanoic acid, methyl ester & $\begin{array}{l}\text { Hexadecanoic acid, methyl ester } \\
\text { ** 6-Octadecenoic acid, methyl ester, (Z)- }\end{array}$ \\
\hline Tridecanoic acid, methyl ester & Tetradecanoic acid, 12-methyl-, methyl ester \\
\hline 2-Pentadecanone, 6,10,14-trimethyl- & $\begin{array}{l}\text { n-Tridecan-1-ol } \\
\text { 1-Tetradecanol } \\
\text { 1-Octadecanol } \\
\text { Octacosanol } \\
\text { Methyl tetracosanoate (Lignoceric acid methyl ester) } \\
\text { Phathalic acid cyclobutyl tridecyl ester } \\
\text { 2-Pentadecanone, 6,10,14-trimethyl- }\end{array}$ \\
\hline 9-Eicosene, $(\mathbf{E})$ - & (Trans)-2-nonadecene \\
\hline \multirow[t]{2}{*}{ 9-Tricosene, (E)- } & 9,12,15-Octadecatrienal \\
\hline & $\begin{array}{l}\text { Decanedioic acid, didecyl ester } \\
\text { Pentadecafluoroctanoic acid octyl ester }\end{array}$ \\
\hline
\end{tabular}




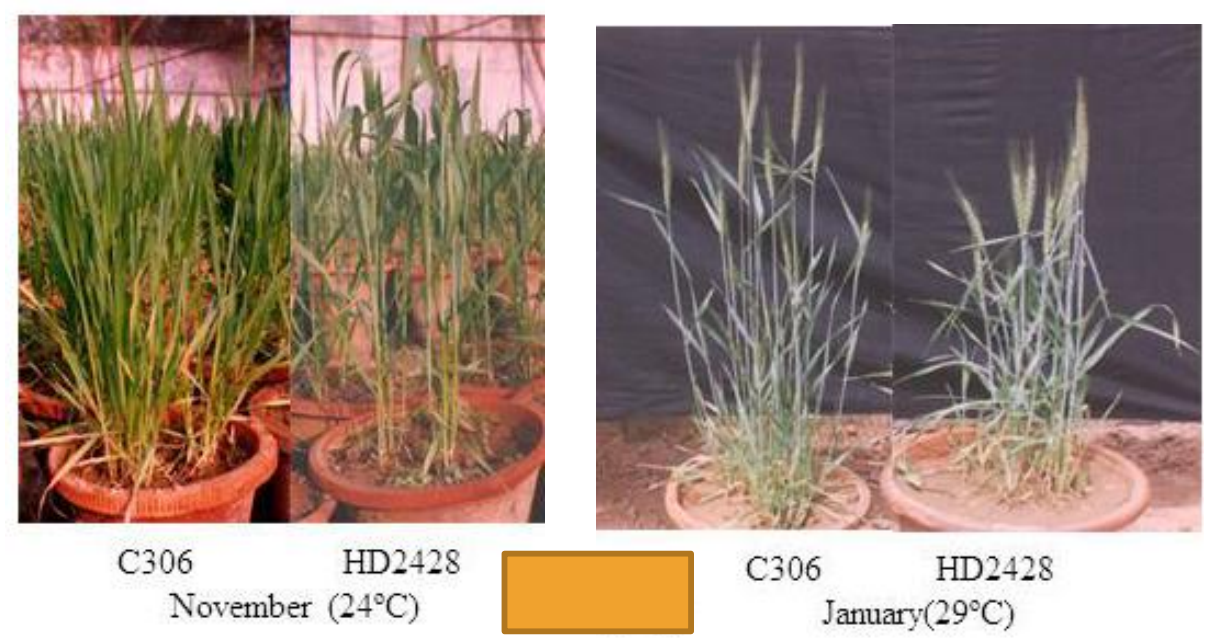

Plate 1. Wax formation on aerial parts of $\mathrm{C306}$ (drought tolerant) and HD2428 (drought sensitive) cultivar of wheat

The phytol is regulated by channelling into tocopherol (vitamin E), phylloquinone (vitamin K) and fatty acid phytyl ester synthesis (Csupor, 1971; Patterson et al., 1993; Ischebeck et al., 2006) containing hexadecatrienoic acid and medium chain fatty acids (10:0, 12:0 and 14:0) in Arabidopsis; palmitate (16:0) in barley and linolenic acid (18:3) in potato and rice . These esters act as transient sink for phytyl and acyl moieties under stress and can be hydrolysed and employed for synthesis of chlorophyll and membrane galactolipids for growth of sink tissues.

Free fatty acids are activated as CoA by chloroplast acyl CoA prior to the phytyl ester synthesis. Phytyl ester (16:3) was most abundant after nitrogen deprivation while (16:0, 18:1 and 18:3) were most abundant after phytol feeding in Arabidopsis. Final catabolism of phytol occurs by $\alpha$ - and $\beta$ oxidation in peroxisomes and mitochondria. On the one hand, phytol and fatty acids accumulation in the phytyl esters represent the limited degradation capacity. On the other hand limitation of two kinases reactions for the conversion of phytol to phytyldiphosphate restrict the channelling of phytol into tocopherol synthesis. Chloroplasts have been shown to harbour the biosynthetic capacity for long chain acyl esters and triacyl glycerols (TAG) in arabidopsis but very long chain fatty acids i.e. octacosanoic (Table 1alcohol) and tetracosanoic acid (Lignoceric acid-accumulation indicate the increased activities of fatty acid elongases at endoplasmic reticulum (ER) in C306 drought tolerant wheat cultivar.

The cuticular waxes are derived from very long chain fatty acids (VLCFA- C20-C34), alkanes, aldehydes, primary and secondary alcohols, ketones and esters but tocopherols, triterpenoids, flavonoids can also form the substantial components. A thick cuticle has been associated with reduced loss of water, therefore, increased resistance/tolerance to water stress (Leide et al., 2007). Drought tolerant cultivar of wheat (C306) exhibits $\mathrm{C} 16+\mathrm{C} 18$ type of fatty acids and more intense wax formation and water retention than drought tolerant wheat cultivar-HD2428 under drought experienced by challenging 
environmental conditions due to high Leide J., Hildebrandt U., Reussing K., radiation and temperature $\left(29^{\circ} \mathrm{C}\right)$. Riederer M., and Vogg, G. (2007). The developmental pattern of tomato fruit

\section{References}

Csupor L. (1971) Das Phytol in vergilbten Blättern. Planta Med. 19: 37-41

Ischebeck T., Zbierzak A. M., Kanwischer M. and Dörmann P. (2006) A salvage pathway for phytol metabolism in Arabidopsis. J. Biol. Chem. 81: 2470 2477.

Kolattukudy P.E. (1980) Biopolyester membranes of plants: cutin and suberin. Science, 208: 990-100. wax accumulation and its impact on cuticular transpiration barrier properties: effects of a deficiency in a $\beta$ ketoacyl-coenzyme A synthase (LeCER6). Plant Physiol. 144: 16671679.

Patterson G., Hugly S. and Harrison D. (1993) Sterols and phytyl esters of Arabidopsis thaliana under normal and chilling temperatures. Phytochemistry 33: 13811383.

\section{How to cite this article:}

Santosh Kumari and Vipin Kumar Verma. 2020. Quality of Wax Esters, Alcohol and Cuticle Formation in Drought Tolerant and Drought Sensitive Wheats (Triticum aestivum) under Unfavourable Temperature and Radiation Conditions for Growth and Development. Int.J.Curr.Microbiol.App.Sci. 9(02): 546-550. doi: https://doi.org/10.20546/ijcmas.2020.902.068 